Case Report

\title{
A Pancreatic Head Tumor Arising as a Duodenal GIST: A Case Report and Review of the Literature
}

\author{
Fabian Bormann, ${ }^{1}$ Wolfgang Wild, ${ }^{1}$ Hüseyin Aksoy, ${ }^{1}$ Pius Dörr, ${ }^{2}$ \\ Sanja Schmeck, ${ }^{3}$ and Matthias Schwarzbach ${ }^{1}$ \\ ${ }^{1}$ Klinik für Allgemein-, Viszeral-, Thorax- und Gefäßchirurgie, Klinikum Frankfurt Höchst, Gotenstraße 6-8, \\ 65929 Frankfurt am Main, Germany \\ ${ }^{2}$ Klinik für Radiologie, Neuroradiologie und Nuklearmedizin, Klinikum Frankfurt Höchst, Gotenstraße 6-8, \\ 65929 Frankfurt am Main, Germany \\ ${ }^{3}$ Institut für Pathologie, Klinikum Frankfurt Höchst, Gotenstraße 6-8, 65929 Frankfurt am Main, Germany
}

Correspondence should be addressed to Fabian Bormann; fabian.bormann@klinikumfrankfurt.de

Received 4 December 2013; Revised 4 May 2014; Accepted 2 July 2014; Published 10 September 2014

Academic Editor: Yasuhiko Sugawara

Copyright (C) 2014 Fabian Bormann et al. This is an open access article distributed under the Creative Commons Attribution License, which permits unrestricted use, distribution, and reproduction in any medium, provided the original work is properly cited.

\begin{abstract}
Gastrointestinal stromal tumors (GISTs) are rare mesenchymal tumors of the gastrointestinal tract that originate from the intestinal cells of Cajal (ICC) (Fletcher et al., 2002). Only a few cases have been described with extragastrointestinal stromal tumors (Kim et al., 2012; Soufi et al., 2013; Meng et al., 2011). They are often diagnosed as a pancreatic head tumor as they are very difficult to relate to the duodenum with CT, MRI, or ultrasound. We present a case of a sixty-four-year-old woman who presented with abdominal pain and cardialgia for a follow-up examination after breast cancer surgery. On laparotomy there was a $3 \times 5 \mathrm{~cm}$ hypervascular mass arising from the pancreatic head with macroscopically no attachment to the duodenum. The patient underwent pancreatoduodenectomy (PD) modified after Traverso-Longmire, histopathology proved a duodenal GIST. This case proves that duodenal GISTs can grow invasively into the pancreas and appear as solid pancreas head tumor; therefore, these tumors should be included into differential diagnosis.
\end{abstract}

\section{Introduction}

GISTs are the most common mesenchymal tumors of the GI [1]. They may arise in any part of the GI but mostly occur in the stomach (60\%) and the small intestine (35\%). Only a few cases have been described with extragastrointestinal stromal tumors [2-4]. They are a very rare tumor identity among the duodenal tumors with less than $5 \%$.

\section{Case Report}

A sixty-four-year-old woman, in good physical condition, with abdominal pain and cardialgia for four weeks was transferred to our clinic. She first presented in the gynecological department for her follow-up examination after a surgical breast cancer treatment nine years ago. The outwards ultrasound of the liver and the upper abdomen showed an unknown formation in the pancreas. The result of the followed endoscopic biopsy showed a carcinoid with an unknown dignity.

In the anamnesis, she described a slowly increasing pain within the last few days, mostly located in the right upper abdomen. She could not tell any relief or aggravation or any kind of radiation. She was not suffering from fever, night sweat, vomiting, jaundice, or weight loss. The history of bowel movement was empty. The physical examination showed a soft abdomen, without any resistance. The pain slightly increased by pressure in the epigastric area. The gallbladder could not be palpated and the liver margin seemed to be inconspicuous. There was no abnormality on digital rectal examination. The routine laboratory tests showed no pathological findings. In our abdominal ultrasound, we found the suspicious heterogeneous formation of $3 \times 5 \mathrm{~cm}$ in the pancreas and a $3 \times 1 \mathrm{~cm}$ cystic formation in liver segment 6 . The CT-scan proved the ultrasound findings and showed the described formation in the pancreatic head, and a connection 

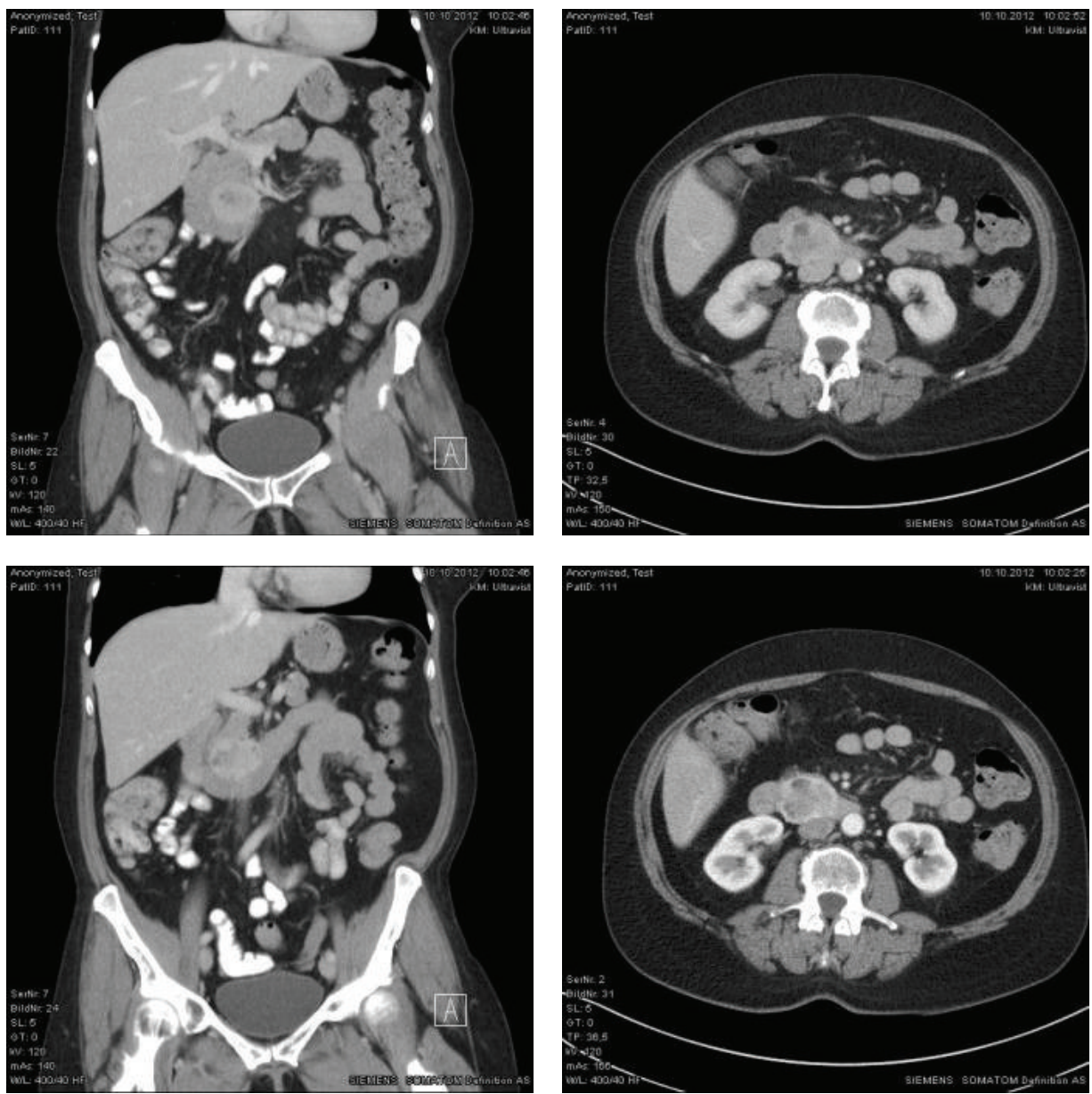

FIgURE 1: CT-scan of the abdomen with the pancreatic mass of enhancing and nonenhancing areas.

to the duodenum could not be found. The well-defined mass of heterogeneous density seemed to consist of enhancing and nonenhancing areas (Figure 1).

In further staging examinations, there was no pathological finding for the tumor markers $C E A, C a-19$ 9, gastrin, and chromogranin. A gastroscopy showed a moderate streaky gastritis of the antrum without any ulcer. The especially for neuroendocrine tumors very sensitive $99 \mathrm{mTc}-\mathrm{EDDA} / \mathrm{HYNIC}-$ TOC scintigraphy did not show any strong enhancement. The tumor could easily be seen by exploratory laparotomy as a hypervascular mass arising from the pancreatic head, which was mobile to the retroperitoneum and seemed to be resectable. It did not infiltrate into the pylorus and macroscopically it did not attach to the duodenum. There was also no peritoneal dissemination. The suspicious liver area was localized and marked by ultrasound. PD modified after Traverso-Longmire was performed and the marked liver area in segment 6 was resected in liver wedge technique.

Histopathology showed a mesenchymal, sharply margined tumor of $4 \mathrm{~cm}$ size, consisting of spindle cells and isolated apoptosis but without necrosis. Mitosis could not be detected. On one side of the tumor, a small connection to the duodenal serosa was conceivable.
In the immunohistology, the tumor was positive for smooth muscle actin (ASMA), bcl-2, CD 99, CD 117, DOG-1, and some cells also for $C D 34$ (Figure 2). Negative staining for desmin and S-100. MIB-1 a marker for proliferation showed a rate of $1 \%$. Further examinations showed an exon 11 mutation in the c-KIT gene.

Based on these findings, the tumor was finally diagnosed as a low risk GIST arising from the duodenal wall, incorporating into the pancreatic head.

Postoperatively the patient suffered from a pneumonia, which was treated with antibiotics. There was no raise of amylase and lipase in the drainage; it was removed at day ten after surgery. The blood glucose was not elevated. The patient was discharged in good condition and advised to take pancreatin beside the meals.

\section{Discussion}

GISTs are quite rare tumors with an incidence of annually only $10-15$ per million [1]. They are the most common mesenchymal tumors of the GI, originating from the ICC, a cell from the autonomic nervous system. For sporadic GISTs, 


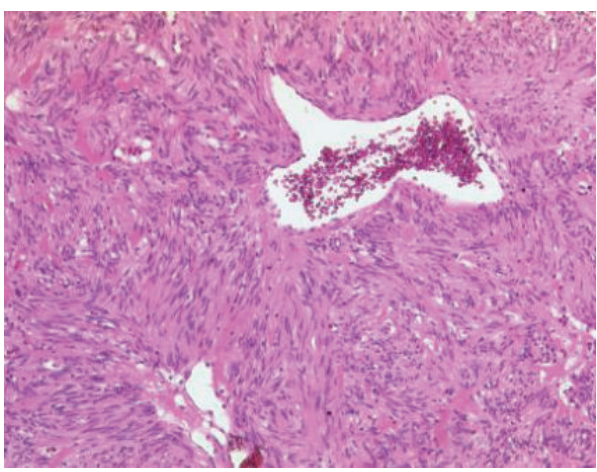

(a)

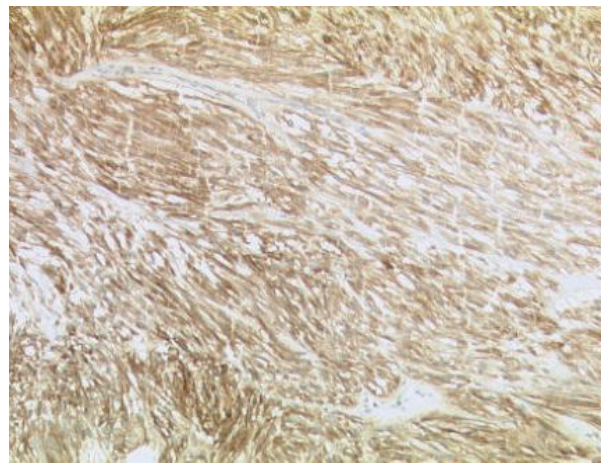

(c)

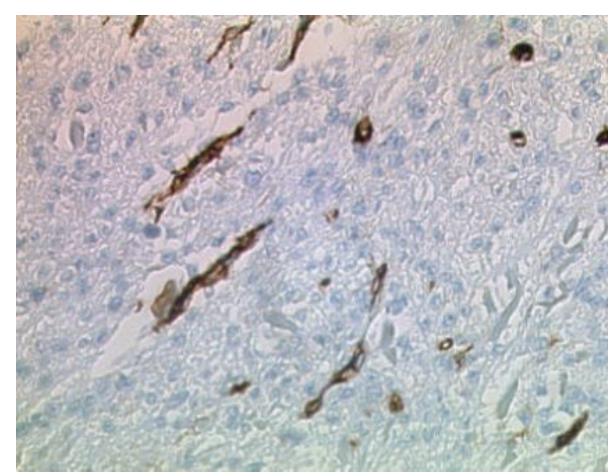

(b)

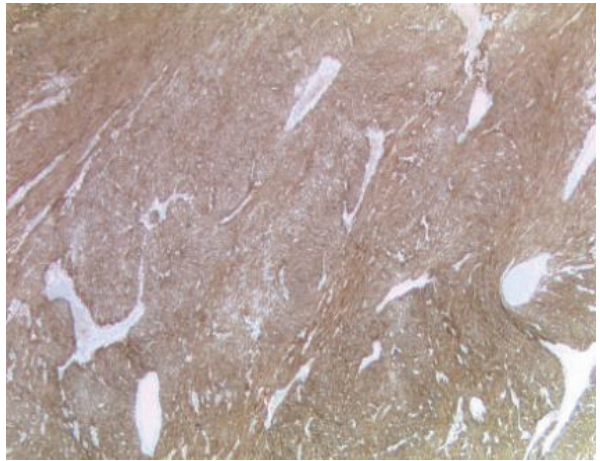

(d)

FIGURE 2: Histopathological pictures of different stainings. (a) Hematoxylin-eosin staining. (b) CD 34 staining. (c) CD117 staining. (d) DOG-1 staining.

there is no gender difference and the median age at the time of diagnosis is 60 years.

GISTs can occur anywhere in the GI but mostly affect the stomach (60\%), jejunum and ileum (30\%) only 4$5 \%$ arise in den duodenum. Depending on the size and location of the tumor, they often present with abdominal discomfort, full feeling, and gastrointestinal bleeding, if the tumor grows invasively into the epithelial layer. At time of diagnosis, approximately $20-50 \%$ of the patients already have metastasis, most likely in the liver and/or the peritoneum. Very rarely can metastasis be found in the lymph nodes; therefore, surgery does not have to be that radical $[5,6]$.

The endoscopy that is often performed by the described symptoms above frequently shows a submucosal bulge or ulceration. Together with an endoscopic ultrasound, the origin can be displayed intra- or extramurally. However, the final diagnosis is proofed by biopsy $[5,7,8]$, which is often performed in a CT or ultrasound guided way. The percutaneous biopsy should only be considered if another differential diagnosis (e.g., lymphoma) or a neoadjuvant treatment is likely. As GISTs are quite fragile and well perfused, it is difficult to perform the biopsy without spreading the tumor cells into the abdominal cavity. With an intraabdominal rupture of the tumor capsule, the development of peritoneal metastasis is almost obligatory. In case of doubt, the tumor should be primarily resected.
GISTs typically express the c-KIT protein (CD 117). Histopathologically the main mechanism in the genesis of a GIST is the mutation in one of two receptor tyrosine kinase genes. Most frequently the mutation is located on KIT exon 11 (70\%), KIT exon 9 (10\%), and PDGFRA exon 18 (5\%). GISTs mostly occur in a spindle cell type (60-70\%) but may also occur in an epithelioid type (20-30\%) or as a mixture. Other typical markers for GISTs are the antibody Discovered on GIST-1 (DOG-1), the cell surface glycoprotein CD34 (70\% are positive), and ASMA ( $40 \%$ are positive). GISTs are mostly negative for desmin and $S-100[5,9,10]$.

If resectable, the tumor should be treated by surgery. Depending on the localization and the size of the tumor, this can be performed laparoscopically or by conventional laparotomy. To be considered as potentially curative, the resection needs to be with a tumor free margin and a safety clearance of at least $1 \mathrm{~cm}[7,8]$.

As in our case described herein, most duodenal GISTs in the pancreas first seem to be a pancreatic head tumor of neuroendocrine origin. In these cases, the surgical resection with tumor free margin is desirable; therefore, we decided to treat with a PD. As there is no benefit in survival for patients with a duodenal GIST treated with PD compared to limited resection (LR), it is very important to optimize the diagnosis to minimize the surgical extent and the morbidity $[11,12]$. 
TABLE 1: Risk classification of duodenal GISTs modified after Miettinen and Lasota [15].

\begin{tabular}{lcc}
\hline Amount of mitosis & Size $(\mathrm{cm})$ & Risk \\
\hline \multirow{3}{*}{$\leq 5$ per $50 \mathrm{HPF}$} & $\leq 2$ & No risk \\
& $2-5$ & Low \\
& $5-10$ & High \\
\hline \multirow{3}{*}{$>5$ per $50 \mathrm{HPF}$} & $\leq 2$ & No data \\
& $2-5$ & High \\
& $5-10$ & High \\
\hline
\end{tabular}

If the tumor is primarily not resectable (size or location) or already with a metastatic spread neoadjuvant chemotherapy for example, with imatinib should be performed. Within 3-4 months, the tumor should be reevaluated and secondly resected $[7,13,14]$.

Regarding the prognosis of malignancy, Miettinen et al. classified by the amount of mitosis per $50 \mathrm{HPF}$ the size and origin of the tumor [15] (Table 1).

Fletcher classified in a similar way [1]. Patients with duodenal GITSs classified as intermediate or high risk for a tumor relapse should be treated with imatinib $400 \mathrm{mg}$ daily for three years. There is no benefit for patients classified at low risk or with a certain PDGFRA mutation (PDGFRA-D842V) [16-18].

The median 5-year survival of resected patient without metastasis is around $50 \%$ without further chemotherapy or biologicals [15, 19-21].

After complete tumor resection, the follow-up care should be within every 3-6 months, including clinical examination and CT-scans of the abdomen and the pelvis once a year for 5 years [16].

\section{Conflict of Interests}

The authors declare that there is no conflict of interests regarding the publication of this paper.

\section{References}

[1] C. D. M. Fletcher, J. J. Berman, C. Corless et al., "Diagnosis of gastrointestinal stromal tumors: a consensus approach," Human Pathology, vol. 33, no. 5, pp. 459-465, 2002.

[2] H.-H. Kim, Y.-S. Koh, E.-K. Park et al., "Primary extragastrointestinal stromal tumor arising in the pancreas: report of a case," Surgery Today, vol. 42, no. 4, pp. 386-390, 2012.

[3] M. Soufi, M. Bouziane, R. Massrouri, and B. Chad, "Pancreatic GIST with pancreas divisum: a new entity," International Journal of Surgery Case Reports, vol. 4, no. 1, pp. 68-71, 2013.

[4] L. Meng, S.-H. Fang, and M. Jin, "An unusual case of pancreatic and gastric neoplasms," European Radiology, vol. 21, no. 3, pp. 663-665, 2011.

[5] M. Miettinen and J. Lasota, "Gastrointestinal stromal tumors: review on morphology, molecular pathology, prognosis, and differential diagnosis," Archives of Pathology and Laboratory Medicine, vol. 130, no. 10, pp. 1466-1478, 2006.
[6] M. R. Hersh, J. Choi, C. Garrett, and R. Clark, "Imaging gastrointestinal stromal tumors," Cancer Control, vol. 12, no. 2, pp. 111-115, 2005.

[7] U. I. Chaudhry and R. P. DeMatteo, "Management of resectable gastrointestinal stromal tumor," Hematology/Oncology Clinics of North America, vol. 23, no. 1, pp. 79-87, 2009.

[8] C. Langer, P. Schüler, H. Becker, and T. Liersch, "Gastrointestinal stromal tumors from the surgical point of view," Chirurg, vol. 79, no. 7, pp. 644-649, 2008.

[9] A. W. Beham, I.-M. Schaefer, P. Schüler, S. Cameron, and B. M. Ghadimi, "Gastrointestinal stromal tumors," International Journal of Colorectal Disease, vol. 27, no. 6, pp. 689-700, 2012.

[10] C. B. Tan, W. Zhi, G. Shahzad, and P. Mustacchia, "Gastrointestinal stromal tumors: a review of case reports, diagnosis treatment and further directions," ISRN Gastroenterology, vol. 2012, Article ID 595968, 16 pages, 2012.

[11] F. M. Johnston, P. J. Kneuertz, J. L. Cameron et al., "Presentation and management of gastrointestinal stromal tumors of the duodenum: a multi-institutional analysis," Annals of Surgical Oncology, vol. 19, no. 11, pp. 3351-3360, 2012.

[12] Y. W. Tien, C. Y. Lee, C. C. Huang, R. H. Hu, and P. H. Lee, "Surgery for gastrointestinal stromal tumors of the duodenum," Annals of Surgical Oncology, vol. 17, no. 1, pp. 109-114, 2010.

[13] C. P. Raut, M. Posner, J. Desai et al., "Surgical management of advanced gastrointestinal stromal tumors after treatment with targeted systemic therapy using kinase inhibitors," Journal of Clinical Oncology, vol. 24, no. 15, pp. 2325-2331, 2006.

[14] A. Gronchi, M. Fiore, F. Miselli et al., "Surgery of residual disease following molecular-targeted therapy with imatinib mesylate in advanced/metastatic GIST," Annals of Surgery, vol. 245, no. 3, pp. 341-346, 2007.

[15] M. Miettinen and J. Lasota, "Gastrointestinal stromal tumors: pathology and prognosis at different sites," Seminars in Diagnostic Pathology, vol. 23, no. 2, pp. 70-83, 2006.

[16] P. G. Casali, L. Jost, P. Reichardt, M. Schlemmer, and J.-Y. Blay, "Gastrointestinal stromal tumours: ESMO Clinical Recommendations for diagnosis, treatment and follow-up," Annals of Oncology, vol. 20, no. 4, pp. iv64-iv67, 2009.

[17] H. Joensuu, M. Eriksson, K. S. Hall et al., "One vs three years of adjuvant imatinib for operable gastrointestinal stromal tumor: a randomized trial," JAMA-Journal of the American Medical Association, vol. 307, no. 12, pp. 1265-1272, 2012.

[18] The ESMO/European Sarcoma Network Working Group, "Gastrointestinal stromal tumors: ESMO clinical practice guidelines for diagnosis, treatment and follow-up," Annals of Oncology, vol. 23, supplement 7, pp. vii49-vii55, 2012.

[19] R. Quek and S. George, "Gastrointestinal stromal tumor: a clinical overview," Hematology/Oncology Clinics of North America, vol. 23, no. 1, pp. 69-78, 2009.

[20] J. Verweij, P. G. Casali, J. Zalcberg et al., "Progression-free survival in gastrointestinal stromal tumours with high-dose imatinib: randomised trial," The Lancet, vol. 364, no. 9440, pp. 1127-1134, 2004.

[21] C. D. Blanke, C. Rankin, G. D. Demetri et al., "Phase III randomized, intergroup trial assessing imatinib mesylate at two dose levels in patients with unresectable or metastatic gastrointestinal stromal tumors expressing the kit receptor tyrosine kinase," Journal of Clinical Oncology, vol. 26, no. 4, pp. 626-632, 2008. 


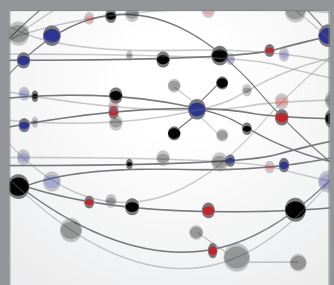

The Scientific World Journal
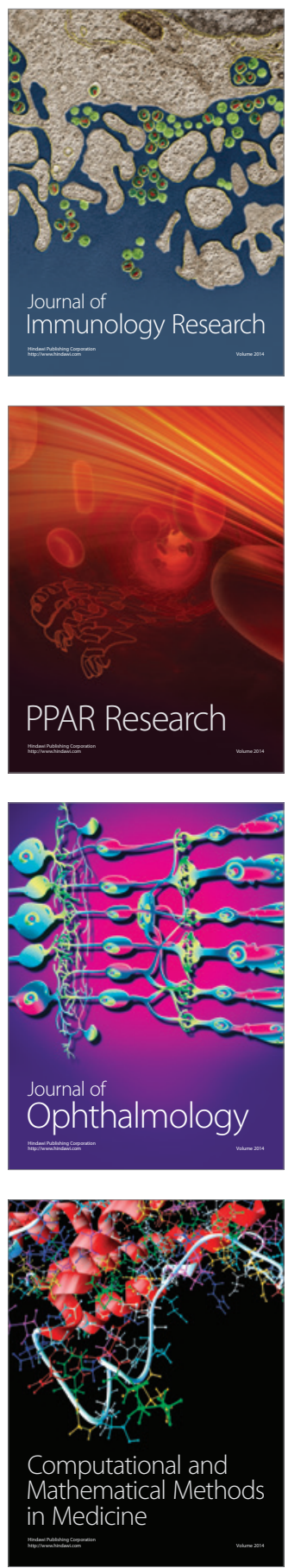

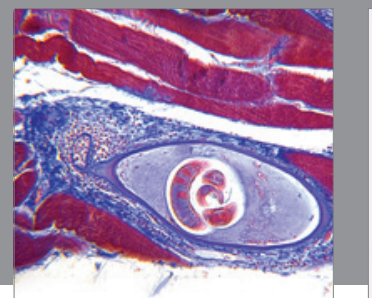

Gastroenterology

Research and Practice
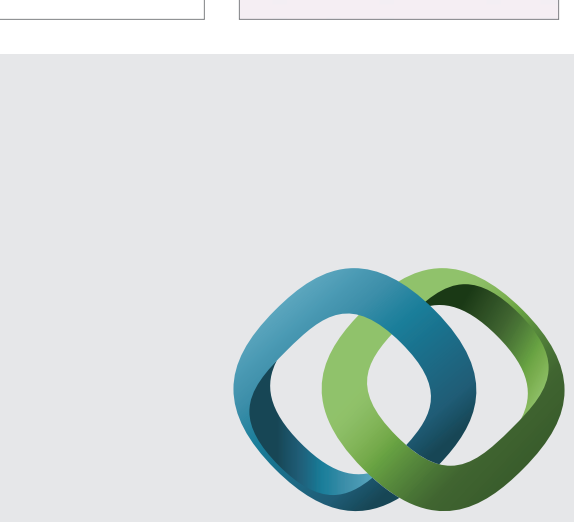

\section{Hindawi}

Submit your manuscripts at

http://www.hindawi.com
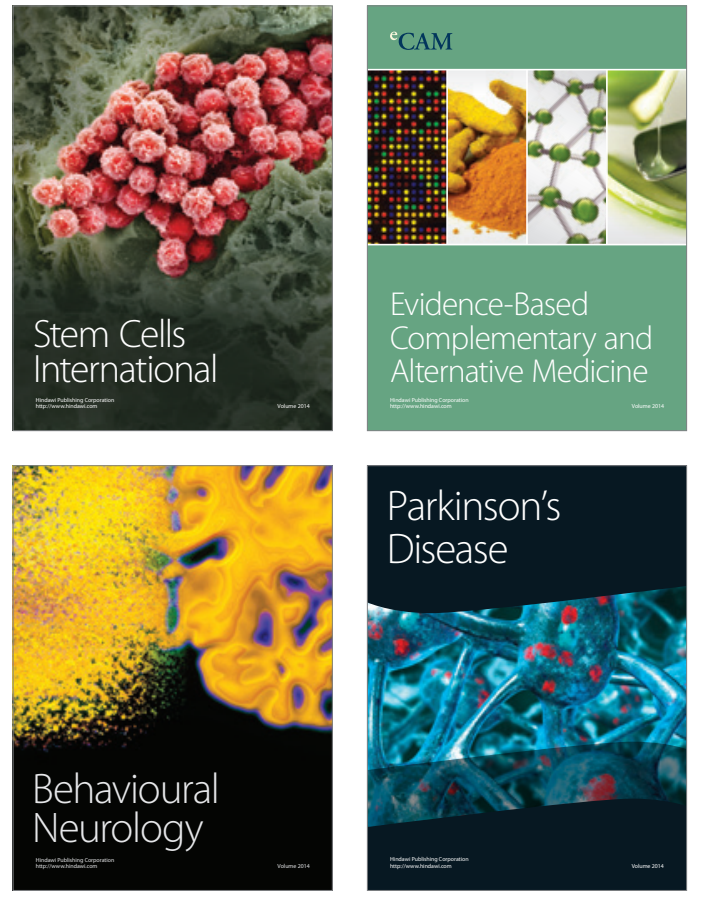
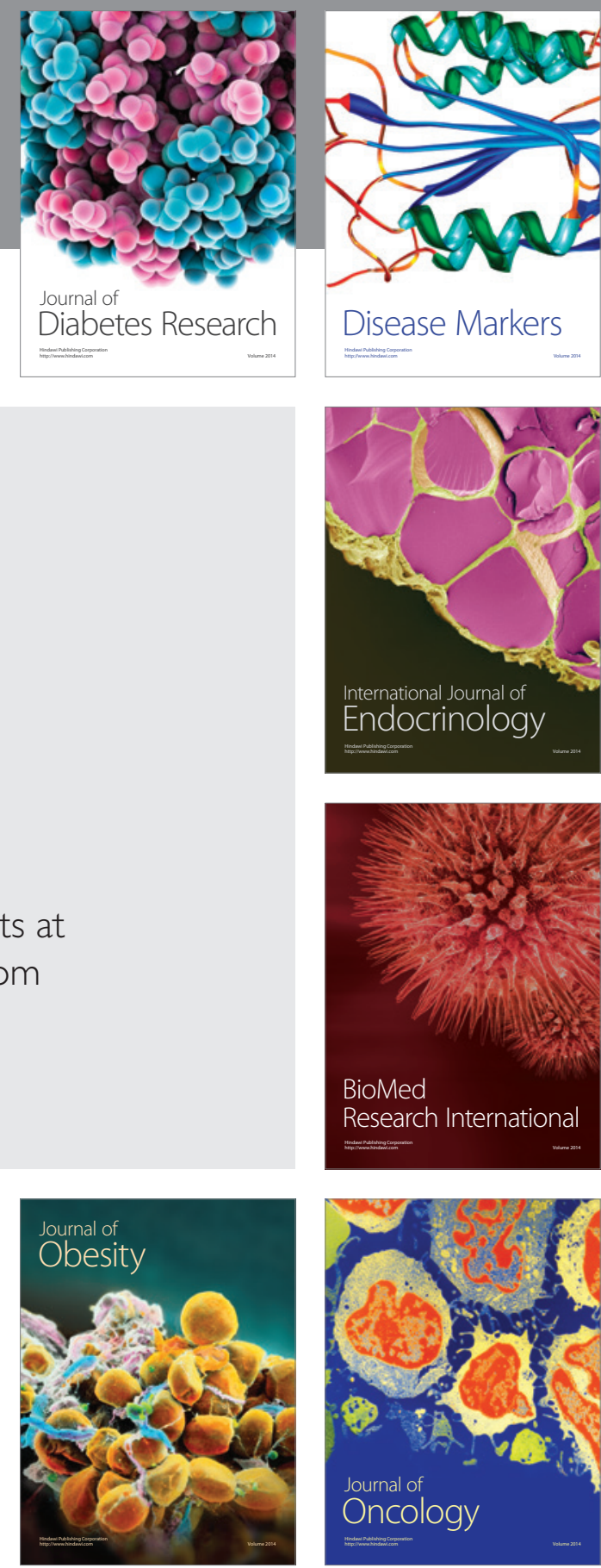

Disease Markers
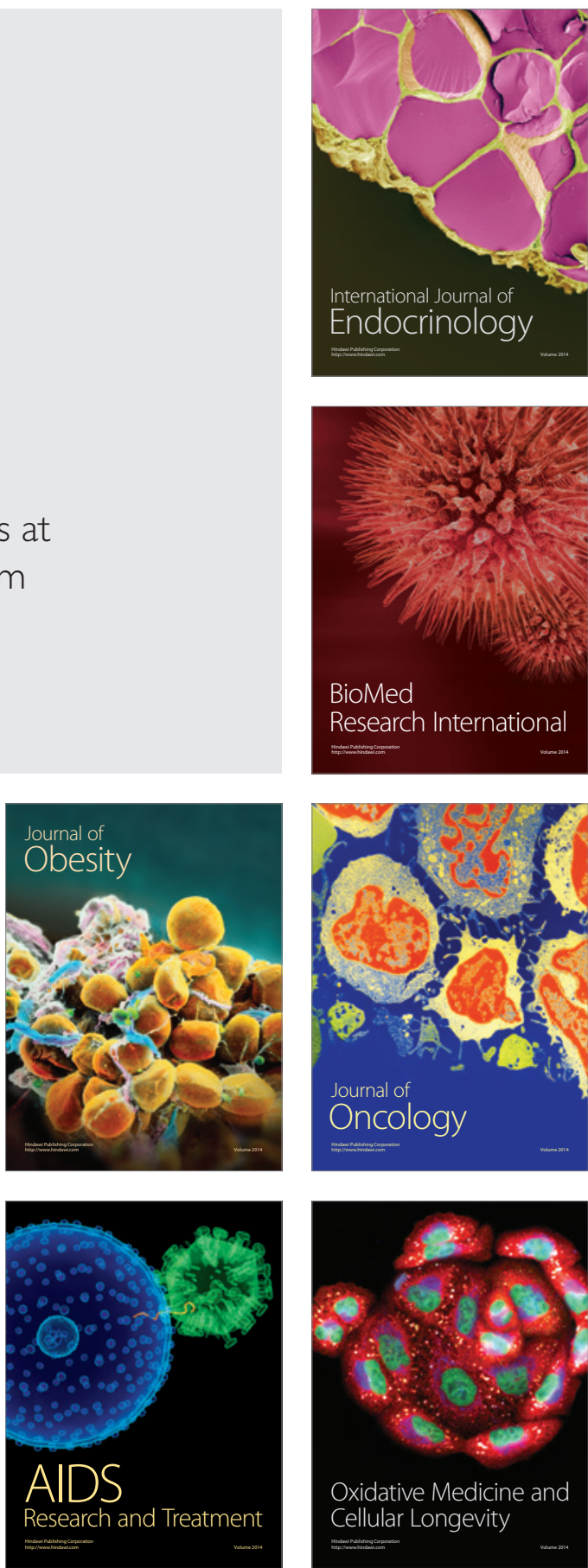